Table S3: Histopathology at 16 weeks of age

Aortic wall thickness

$\begin{array}{llrrrr}\text { Genotype } & \text { Intervention } & \text { Mean } & \text { SD } & \boldsymbol{P} \text { vs } \boldsymbol{W T} \text { - } \boldsymbol{N} \boldsymbol{T} & \boldsymbol{P} \text { vs DD-NT } \\ \text { WT } & \text { NT } & 61.61 & 6.98 & & \\ \text { WT } & \text { LP } & 57.84 & 10.17 & 1.000 & \\ \text { WT } & \text { LN } & 58.88 & 8.44 & 1.000 & \\ \text { WT } & \text { AP } & 64.91 & 10.34 & 1.000 & \\ \text { WT } & \text { AN } & 61.50 & 4.44 & 1.000 & \\ \text { DD } & \text { NT } & 77.61 & 5.70 & \mathbf{0 . 0 0 0} & \\ \text { DD } & \text { LP } & 58.39 & 18.43 & 1.000 & \mathbf{0 . 0 0 8} \\ \text { DD } & \text { LN } & 68.02 & 2.19 & 0.376 & 1.000 \\ \text { DD } & \text { AP } & 70.74 & 8.06 & 0.069 & 1.000 \\ \text { DD } & \text { AN } & 72.11 & 8.64 & 0.078 & 1.000 \\ \text { DD/Ncf1- } & \text { NT } & 68.69 & 9.29 & \mathbf{0 . 0 3 5} & \mathbf{0 . 0 0 7} \\ \text { DD/Ncf1- } & \text { LP } & 62.64 & 10.35 & 1.000 & \mathbf{0 . 0 0 2} \\ \text { DD/Ncf1- } & \text { LN } & 68.72 & 5.23 & 0.125 & \mathbf{0 . 0 3 1} \\ \text { DD/Ncf1- } & \text { AP } & 63.46 & 3.25 & 1.000 & \mathbf{0 . 0 0 0} \\ \text { DD/Ncf1- } & \text { AN } & 65.19 & 10.65 & 1.000 & \mathbf{0 . 0 2 2}\end{array}$

\title{
Number of lamellar units
}

$\begin{array}{lrrr}\text { Genotype } & \text { Mean } & \text { SD } & \boldsymbol{P} \text { vs } \boldsymbol{W T} \text {-NT } \\ \text { WT } & 7.12 & 0.70 & \\ \text { DD } & 8.39 & 1.38 & \mathbf{0 . 0 1 6} \\ \text { DD/Ncf1- } & 7.85 & 1.31 & 0.252\end{array}$

\% Heart weight / Body weight

\begin{tabular}{llrlll}
\multicolumn{2}{c}{ Genotype } & Intervention & Mean & SD $\boldsymbol{P}$ vs $\boldsymbol{W T}$ - $\boldsymbol{N} \boldsymbol{T}$ & $\boldsymbol{P}$ vs $\boldsymbol{D} \boldsymbol{D}-\boldsymbol{N} \boldsymbol{T}$ \\
WT & NT & 0.56 & 0.11 & & \\
WT & LP & 0.52 & 0.07 & 1.000 & \\
WT & LN & 0.46 & 0.07 & 0.913 & \\
WT & AP & 0.60 & 0.09 & 1.000 & \\
WT & AN & 0.54 & 0.11 & 1.000 & \\
DD & NT & 0.89 & 0.11 & $\mathbf{0 . 0 0 0}$ & \\
DD & LP & 0.65 & 0.09 & 0.716 & $\mathbf{0 . 0 0 1}$ \\
DD & LN & 0.54 & 0.04 & 1.000 & $\mathbf{0 . 0 0 0}$ \\
DD & AP & 0.69 & 0.09 & 0.145 & $\mathbf{0 . 0 0 2}$ \\
DD & AN & 0.73 & 0.02 & 0.291 & $\mathbf{0 . 0 3 1}$ \\
DD/Ncf1- & NT & 0.74 & 0.08 & $\mathbf{0 . 0 4 6}$ & $\mathbf{0 . 0 3 9}$ \\
DD/Ncf1- & LP & 0.48 & 0.10 & 0.737 & $\mathbf{0 . 0 0 0}$ \\
DD/Ncf1- & LN & 0.56 & 0.07 & 1.000 & $\mathbf{0 . 0 0 0}$ \\
DD/Ncf1- & AP & 0.54 & 0.07 & 1.000 & $\mathbf{0 . 0 0 0}$ \\
DD/Ncf1- & AN & 0.61 & 0.16 & 1.000 & $\mathbf{0 . 0 0 3}$
\end{tabular}


Table S3: Histopathology at 16 weeks of age

Cross-sectional area of cardiomyocytes $\left(\mu \mathrm{m}^{2}\right)$

Left Ventricle (LV)

$\begin{array}{llrrrr}\text { Genotype } & \text { Intervention } & \text { Mean } & \text { SD } & \boldsymbol{P} \text { vs WT-NT } & \boldsymbol{P} \text { vs DD-NT } \\ \text { WT } & \text { NT } & 240.70 & 17.70 & & \\ \text { WT } & \text { LP } & 257.65 & 33.47 & 1.000 & \\ \text { WT } & \text { LN } & 253.04 & 23.61 & 1.000 & \\ \text { WT } & \text { AP } & 261.95 & 32.80 & 1.000 & \\ \text { WT } & \text { AN } & 260.32 & 33.78 & 1.000 & \\ \text { DD } & \text { NT } & 313.52 & 2.12 & \mathbf{0 . 0 0 0} & \\ \text { DD } & \text { LP } & 260.62 & 26.52 & 0.517 & \mathbf{0 . 0 0 6} \\ \text { DD } & \text { LN } & 271.63 & 29.57 & 0.080 & \mathbf{0 . 0 4 6} \\ \text { DD } & \text { AP } & 267.86 & 23.09 & 0.096 & \mathbf{0 . 0 2 3} \\ \text { DD } & \text { AN } & 266.17 & 24.60 & 0.088 & \mathbf{0 . 0 1 7} \\ \text { DD/Ncf1- } & \text { NT } & 294.38 & 6.17 & \mathbf{0 . 0 0 0} & \mathbf{0 . 0 2 5} \\ \text { DD/Ncf1- } & \text { LP } & 246.33 & 26.87 & 1.000 & \mathbf{0 . 0 0 0} \\ \text { DD/Ncf1- } & \text { LN } & 261.69 & 15.42 & 0.348 & \mathbf{0 . 0 0 0} \\ \text { DD/Ncf1- } & \text { AP } & 269.80 & 18.48 & 0.069 & \mathbf{0 . 0 0 0} \\ \text { DD/Ncf1- } & \text { AN } & 261.59 & 9.43 & 0.201 & \mathbf{0 . 0 0 0}\end{array}$

Right Ventricle (RV)

$\begin{array}{llrrrr}\text { Genotype } & \text { Intervention } & \text { Mean } & \text { SD } & \boldsymbol{P} \text { vs } \boldsymbol{W T} \text {-NT } & \boldsymbol{P} \text { vs DD-NT } \\ \text { WT } & \text { NT } & 322.08 & 18.04 & & \\ \text { WT } & \text { LP } & 315.12 & 25.12 & 1.000 & \\ \text { WT } & \text { LN } & 327.55 & 27.74 & 1.000 & \\ \text { WT } & \text { AP } & 326.17 & 21.67 & 1.000 & \\ \text { WT } & \text { AN } & 309.57 & 17.58 & 1.000 & \\ \text { DD } & \text { NT } & 433.29 & 20.24 & \mathbf{0 . 0 0 0} & \\ \text { DD } & \text { LP } & 344.02 & 11.22 & 0.247 & \mathbf{0 . 0 0 0} \\ \text { DD } & \text { LN } & 340.09 & 35.21 & 0.895 & \mathbf{0 . 0 0 0} \\ \text { DD } & \text { AP } & 364.89 & 38.51 & \mathbf{0 . 0 3 1} & \mathbf{0 . 0 0 2} \\ \text { DD } & \text { AN } & 351.54 & 18.35 & 0.100 & \mathbf{0 . 0 0 0} \\ \text { DD/Ncf1- } & \text { NT } & 407.02 & 6.74 & \mathbf{0 . 0 0 0} & \mathbf{0 . 0 3 9} \\ \text { DD/Ncf1- } & \text { LP } & 331.18 & 28.27 & 1.000 & \mathbf{0 . 0 0 0} \\ \text { DD/Ncf1- } & \text { LN } & 327.52 & 30.79 & 1.000 & \mathbf{0 . 0 0 0} \\ \text { DD/Ncf1- } & \text { AP } & 337.36 & 10.67 & 0.938 & \mathbf{0 . 0 0 0} \\ \text { DD/Ncf1- } & \text { AN } & 330.09 & 27.54 & 1.000 & \mathbf{0 . 0 0 0}\end{array}$

